\title{
Utility of Clinical Risk Stratification in the Selection of Muscle-Invasive Bladder Cancer Patients for Neoadjuvant Chemotherapy: A Retrospective Cohort Study
}

Friedrich-Carl von Rundstedt ${ }^{\mathrm{a}, \mathrm{b}, 1}$, Douglas A. Mata ${ }^{\mathrm{c}, 1}$, Oleksandr N. Kryvenko ${ }^{\mathrm{d}}$, Anup A. Shah ${ }^{\mathrm{e}}$, Iny Jhun ${ }^{\mathrm{c}}$ and Seth P. Lerner ${ }^{\mathrm{a}, *}$

${ }^{a}$ Scott Department of Urology, Baylor College of Medicine, Houston, TX, USA

${ }^{\mathrm{b}}$ Department of Urology, Jena University Hospital, Friedrich-Schiller University, Jena, Germany

'Department of Pathology, Brigham and Women's Hospital, Harvard Medical School, Boston, MA, USA

${ }^{\mathrm{d}}$ Departments of Pathology and Urology, Sylvester Comprehensive Cancer Center, University of Miami Miller School of Medicine, FL, USA

${ }^{\mathrm{e}}$ Department of Urology, University of Pittsburgh Medical Center, PA, USA

\begin{abstract}
.
Introduction: Level I evidence supports the use of cisplatin-based neoadjuvant chemotherapy (NAC) for muscle-invasive bladder cancer prior to radical cystectomy (RC). On average, $30-40 \%$ of patients achieve a complete pathologic response (i.e., stage pT0) after receiving NAC. Some centers risk-stratify patients, suggesting that there may be a higher-risk population that would derive the most benefit from NAC. Recently, a risk-stratification model developed at M.D. Anderson Cancer Center (MDACC) specified criteria for clinical staging and patient selection for NAC. We applied this model to our own RC patient cohort and evaluated our own experience with clinical risk stratification and the effect of NAC on post treatment risk categories.

Methods: We retrospectively reviewed the charts of consecutive patients who underwent RC at two institutions between 2004 and 2014 and noted whether or not they received NAC. We determined the clinical stage by reviewing the exam under anesthesia, transurethral resection biopsy (TURBT) pathology, and preoperative imaging. Patients with cT2-T4a nodenegative disease were included. Those with sarcomatoid features or adenocarcinoma were excluded. Patients were classified as high risk if they had tumor-associated hydronephrosis, clinical stage $\geq \mathrm{T} 3 \mathrm{~b}$-T4a disease, variant histology (i.e., micropapillary or small cell), or lymphovascular invasion (LVI), as specified by the MDACC model. Variables were examined for associations with cancer-specific survival (CSS), overall survival (OS), and risk-category reclassification.

Results: We identified 166 patients with a median follow-up time of 22.2 months. In all, 117 patients $(70.5 \%)$ did not receive NAC, $68(58.1 \%)$ of whom we classified as high risk. Among patients not receiving NAC, CSS and OS were significantly decreased in high-risk patients (log-rank test $p=0.01$ for both comparisons). The estimated age-adjusted hazard ratios of highrisk classification for cancer-specific and overall death were 3.2 (95\% CI: 1.2 to 8.6) and 2.2 (95\% CI: 1.1 to 4.4), respectively.
\end{abstract}

\footnotetext{
${ }^{1}$ FCvR and DAM contributed equally to this report.

${ }^{*}$ Correspondence to: Seth P. Lerner, MD, FACS, Beth and Dave Swalm Chair in Urologic Oncology, Professor of Urology, Scott
}

Department of Urology, Baylor College of Medicine Medical Center, 7200 Cambridge, MC BCM380, A10.107, Houston, TX 77030, USA. Tel.: +1 713798 6841; E-mail: slerner@bcm.edu. 
On post-RC final pathology, 23 (46.9\%) low-risk patients were up-classified to high risk and 17 (25.0\%) high-risk patients were down-classified. Complete pathologic responses (pT0) were achieved in $7(6.0 \%)$ patients and partial responses (pT1, pTa, pTis) were achieved in 28 (23.9\%) patients. Of the 49 patients who did receive NAC, 43 (87.8\%) received cisplatin-based and six (12.2\%) received carboplatin-based regimens. Applying the MDACC model, we categorized 41 (83.7\%) patients as high risk prior to NAC treatment. On final pathology, $3(37.5 \%)$ low-risk patients were up-classified and 17 (41.5\%) high-risk patients were down-classified. Complete pathologic responses (pT0) were seen in $13(26.5 \%)$ patients and partial responses were seen in $10(20.4 \%)$ patients. Although the utilization of NAC was not statistically significantly associated with CSS or OS (log-rank test $p>0.05$ for both comparisons), it was associated with a 1.2 times increased odds (95\% CI: 0.4 to 2.1 ) of post-RC reclassification from high to low risk on age-adjusted logistic regression.

Conclusions: We found similar results using the clinical risk-stratification model in our cohort and showed that the high-risk category was associated with lower CSS and OS. NAC was associated with a higher probability of risk reclassification from high to low risk.

Keywords: bladder cancer, neoadjuvant chemotherapy, clinical staging, risk stratification

\section{INTRODUCTION}

The evidence for recommending neoadjuvant chemotherapy (NAC) for muscle-invasive bladder cancer prior to radical cystectomy (RC) is based on four prospective clinical trials [1-4]. The European Organization for Research and Treatment of Cancer/Medical Research Council Advanced Bladder Cancer Working Party (EORTC/MRC) trial demonstrated an absolute long-term survival benefit of approximately $6 \%$ with a relative risk improvement of death of $16 \%$ at ten years [5]. According to a recent meta-analysis, $28.6 \%$ of patients achieve a pT0 stage after NAC [6]. Patients in the NAC arm of the SWOG 8710 trial had more favorable disease-free and overall survivals, and the advantage was most pronounced for patients with clinical T3-T4a disease [1]. This was the rationale for the SWOG S0219 trial that examined clinical and pathologic $\mathrm{T} 0$ rates after neoadjuvant chemotherapy with paclitaxel, carboplatin, and gemcitabine and transurethral resection of bladder tumor (TURBT) [7]. While cT0 rates were as high as $46 \%$, the primary endpoint was not met, as $60 \%$ of those patients had residual cancer at RC. The trial provided additional evidence of the limitations of clinical staging based on TURBT.

The utilization of high-risk features to select patients for NAC or adjuvant chemotherapy was first reported by Millikan et al. in their prospective trial of methotrexate, vinblastine, doxorubicin, and cisplatin (MVAC) chemotherapy [8]. They included a threedimensional mass on exam under anesthesia (EUA), involvement of adjacent organs (e.g., prostatic stromal invasion on transurethral biopsy of the prostatic urethra or direct invasion into the vagina), and the presence of lymphovascular invasion (LVI). Culp et al. expanded on this in order to determine which patients would benefit the most from NAC prior to RC. They classified patients with hydronephrosis, clinical T3b-T4a disease, LVI, or aberrant histology (i.e., micropapillary or neuroendocrine/small cell features) as high risk, and classified those without such features as low risk. They found that $49 \%$ of lowrisk patients were up-classified to high risk after RC [9]. Nevertheless, these patients still had a superior outcome compared to patients who were classified as high risk both before and after RC. Their results were validated in a cohort from the University of Southern California that included 1,138 low-risk and 644 highrisk patients. The study showed consistent results in staging and similar percentages of high-risk patients in the MDACC and USC cohorts as well as similar five-year survival probabilities.

The aim of the present study was to describe our own experience with clinical risk stratification, to validate and potentially refine the MDACC model, and to examine the effect of NAC on clinical risk criteria in our RC cohort.

\section{MATERIALS AND METHODS}

\section{Study participants}

We retrospectively reviewed the charts of consecutive patients who underwent RC with pelvic lymphadenectomy (PLND) at Baylor St. Luke's Medical Center and The Methodist Hospital in Houston, Texas between 2004 and 2014 using a prospective database approved by the institutional review boards of both institutions. We examined operative, pathology, and imaging reports and recorded patient 
demographic information and whether or not patients received NAC. We determined the clinical stage by reviewing the EUA (if available in the operative note), the TURBT, the pathologic evaluation, and preoperative cross-sectional imaging. Imaging findings such as bladder-wall thickening and invasion into perivesical fat or adjacent organs were obtained from the radiology report. Patients were included in the study if they had cT2-T4a node-negative disease. Patients with sarcomatoid features or adenocarcinoma on TURBT were excluded as such patients are not typically treated with chemotherapy and require different interventions. Furthermore, these histologic features were not included in the original analysis by Culp et al. [9].

\section{Risk stratification}

We classified patients as either high or low risk according to the method previously described by Culp et al. at MDACC. Patients were considered high risk if they had tumor-associated hydronephrosis, clinical stage $\geq \mathrm{T} 3 \mathrm{~b}-\mathrm{T} 4 \mathrm{a}$ disease, aberrant histology, or LVI on pathologic evaluation of the TURBT specimen. Hydronephrosis was evaluated based on preoperative imaging and the operative findings at the time of TURBT. If a tumor covered the orifice but did not invade the trigone or the intramural ureter it was not considered hydronephrosis. We did not include cases of hydronephrosis due to separate intra-ureteral lesions. Clinical stage $\geq \mathrm{T} 3 \mathrm{~b}-\mathrm{T} 4 \mathrm{a}$ disease was considered to be present if the EUA revealed a palpable three-dimensional mass or invasion of adjacent structures, TURBT indicated prostatic stromal invasion, or when imaging indicated the presence of marked bladder-wall thickening or possible invasion into the adjacent pelvic organs.

Aberrant histology was designated as the presence of any micropapillary or small-cell carcinoma regardless of the percentage of the non-urothelial tumor component. Immunohistochemistry for neuroendocrine markers (e.g., CD56, synaptophysin, chromogranin A) was used when needed to confirm the diagnosis of small-cell carcinoma (Fig. 1). Urothelial carcinoma with squamous or glandular differentiation was not considered a high-risk feature, as in stage-matched cases these two histologic patterns do not impact prognosis [10]. LVI was defined as the presence of tumor cells within an endotheliumlined space. Immunohistochemistry was not routinely performed to confirm or identify LVI.

\section{Statistical analyses}

Clinical factors were explored using descriptive statistics and examined for associations with cancerspecific survival (CSS), overall survival (OS), and risk-category reclassification. Upstaging after RC was defined as having a pathologic $\mathrm{T}$ stage higher than the clinical $\mathrm{T}$ stage or any $\mathrm{N}+$. In the primary analysis we included all patients who did not receive NAC for validation of the MDACC clinical risk-stratification model. In the secondary analysis we focused on patients who had received NAC and examined the effect of NAC on clinical risk stratification and survival. Differences in sample medians were assessed using the Wilcoxon-Mann-Whitney test; those among categorical variables were assessed using Fisher's exact test. Variables were examined in Cox proportional hazards regression models for associations with CSS and OS. CSS was the time from $\mathrm{RC}$ to the time of disease-related death. OS was the time from $\mathrm{RC}$ to time of death due to any cause. Statistical tests were two-sided and used a significance threshold of $p<0.05$. Reported $p$ values were not adjusted for multiple testing. All analyses were performed using $\mathrm{R}$ version 3.2.3 ( $\mathrm{R}$ Foundation for Statistical Computing, Vienna, Austria).

\section{RESULTS}

\section{Study participants}

We identified 256 patients who underwent $\mathrm{RC}$ with PLND between 2004 and 2014 for all clinical stages (Fig. 2). In all, 166 patients with $\geq$ cT2-T4N0 disease and complete clinical records were included in the analysis (Table 1). Of these patients, $117(70.5 \%)$ did not receive NAC and 49 (29.5\%) did receive NAC. The median follow-up time for the entire cohort was 22.2 months (IQR 10.6 to 46.1).

\section{Risk classification of patients who did not receive NAC}

We risk stratified the 117 patients who did not receive NAC prior to $\mathrm{RC}$ according to the previously published MDACC risk-stratification model. Sixty-eight $(58.1 \%)$ of the patients were classified as high risk and 49 (41.9\%) were classified as low risk. CSS and OS were significantly decreased in the high-risk patient population (log-rank test $p=0.01$ for both comparisons) (Fig. 3). The estimated ageadjusted hazard ratios of high-risk classification for 

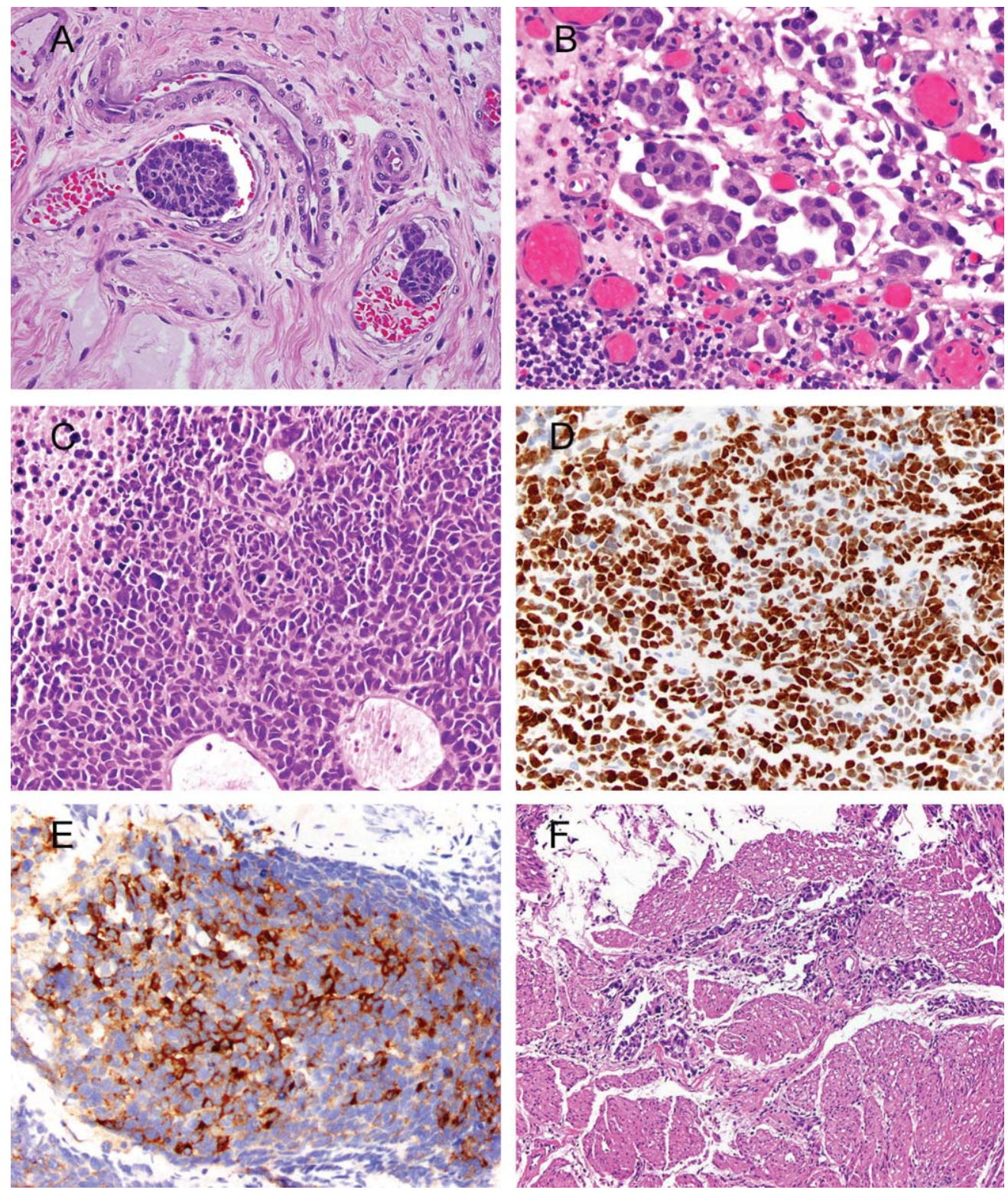

Fig. 1. Photomicrographs illustrating histopathologic features that are important for clinical risk stratification. A. Lymphovascular invasion; H\&E, 40x. Cancer emboli are seen in two vessels. Spaces surrounding cancer cells are disproportional by size and shape, have proteinaceous material and red blood cells, and are lined by endothelium. B. Micropapillary invasive urothelial carcinoma; H\&E, 40x. Lacunae with multiple micropapillae are considered to be the most reproducible microscopic feature. C. Small-cell carcinoma; H\&E, 40x. Solid sheets of high grade cells with scant cytoplasm, frequent mitoses, apoptotic bodies, and necrosis. D. The Ki- 67 nuclear labeling index is $>90 \%$ in small-cell carcinoma; 40x. E. Positive immunostaining for synaptophysin in small-cell carcinoma; 40x. F. Urothelial carcinoma invading thick bundles of smooth muscle diagnostic of detrusor-muscle invasion at transurethral resection.

cancer-specific and overall death were $3.2(95 \% \mathrm{CI}$ : 1.2 to 8.6 ) and 2.2 (95\% CI: 1.1 to 4.4$)$, respectively. On final pathology after RC, 26 (53.1\%) low-risk patients remained classified as such, and $23(46.9 \%)$ were up-classified to high risk. Seventeen $(25.0 \%)$ high-risk patients were down-classified to low-risk and $51(75.0 \%)$ remained high risk (Table 2). In the low-risk category complete pathologic responses (pT0) were achieved in $5(10.2 \%)$ patients and partial responses (pT1, pTa, pTis) were achieved in $16(32.7 \%)$ patients. In the high-risk category complete pathologic responses (pT0) were achieved in 
Underwent radical cystectomy and pelvic

lymphadenectomy between 2004-2014 $(n=256)$

Excluded $(n=90)$

cT2-T4, node-negative disease $(n=166)$

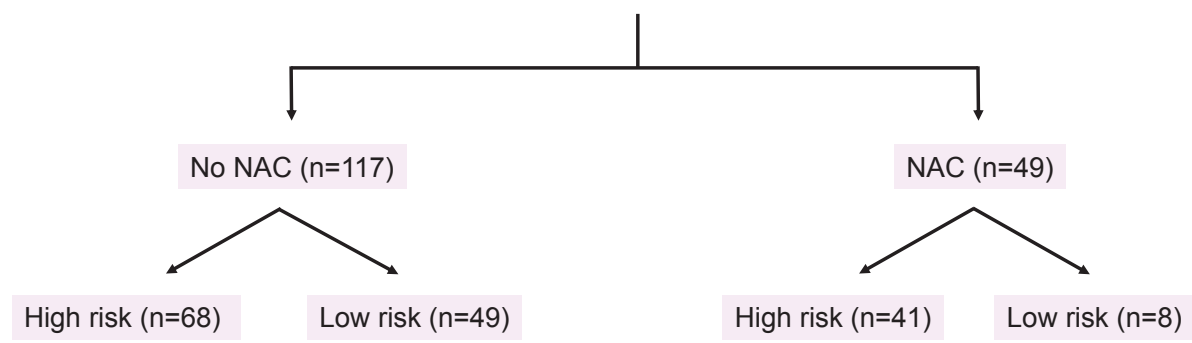

Fig. 2. Study flow diagram.

Table 1

Demographic and clinical characteristics of patients included in the study cohort

\begin{tabular}{|c|c|c|c|c|c|c|c|c|c|c|c|c|}
\hline & \multicolumn{6}{|c|}{ No NAC $(n=117)$} & \multicolumn{6}{|c|}{ NAC $(n=49)$} \\
\hline & \multicolumn{2}{|c|}{ High Risk } & \multicolumn{2}{|c|}{ Low Risk } & \multirow{2}{*}{$\frac{\text { Total }}{\mathrm{n}}$} & \multirow[t]{2}{*}{$P$ value } & \multicolumn{2}{|c|}{ High Risk } & \multicolumn{2}{|c|}{ Low Risk } & \multirow{2}{*}{$\frac{\text { Total }}{\mathrm{n}}$} & \multirow[t]{2}{*}{$P$ value } \\
\hline & $\mathrm{n}$ & $\%$ & $\mathrm{n}$ & $\%$ & & & $\mathrm{n}$ & $\%$ & $\mathrm{n}$ & $\%$ & & \\
\hline \multicolumn{13}{|l|}{$\operatorname{Sex}$} \\
\hline Female & 14 & $(82.4 \%)$ & 3 & $(17.6 \%)$ & 17 & 0.03 & 6 & $(100 \%)$ & 0 & $(0 \%)$ & 6 & 0.25 \\
\hline Male & 54 & $(54 \%)$ & 46 & $(46 \%)$ & 100 & & 35 & $(81.4 \%)$ & 8 & $(18.6 \%)$ & 43 & \\
\hline \multicolumn{13}{|l|}{ Ethnicity } \\
\hline White & 61 & $(59.2 \%)$ & 42 & $(40.8 \%)$ & 103 & 0.82 & 38 & $(82.6 \%)$ & 8 & $(17.4 \%)$ & 46 & 0.73 \\
\hline Black & 2 & $(50 \%)$ & 2 & $(50 \%)$ & 4 & & 1 & $(100 \%)$ & 0 & $(0 \%)$ & 1 & \\
\hline Hispanic & 3 & $(42.9 \%)$ & 4 & $(57.1 \%)$ & 7 & & 2 & $(100 \%)$ & 0 & $(0 \%)$ & 2 & \\
\hline Not reported & 2 & $(66.7 \%)$ & 1 & $(33.3 \%)$ & 3 & & 0 & $(0 \%)$ & 0 & $(0 \%)$ & 0 & \\
\hline \multicolumn{13}{|l|}{ Hydronephrosis } \\
\hline No & 35 & $(41.7 \%)$ & 49 & $(58.3 \%)$ & 84 & $<0.001$ & 24 & $(75 \%)$ & 8 & $(25 \%)$ & 32 & 0.02 \\
\hline Yes & 33 & $(100 \%)$ & 0 & $(0 \%)$ & 33 & & 17 & $(100 \%)$ & 0 & $(0 \%)$ & 17 & \\
\hline \multicolumn{13}{|c|}{ Lymphovascular invasion } \\
\hline No & 46 & $(48.4 \%)$ & 49 & $(51.6 \%)$ & 95 & $<0.001$ & 26 & $(76.5 \%)$ & 8 & $(23.5 \%)$ & 34 & 0.04 \\
\hline Yes & 22 & $(100 \%)$ & 0 & $(0 \%)$ & 22 & & 15 & $(100 \%)$ & 0 & $(0 \%)$ & 15 & \\
\hline \multicolumn{13}{|c|}{ Aberrant histology } \\
\hline No & 62 & $(55.9 \%)$ & 49 & $(44.1 \%)$ & 111 & 0.03 & 27 & $(77.1 \%)$ & 8 & $(22.9 \%)$ & 35 & 0.05 \\
\hline Yes & 6 & $(100 \%)$ & 0 & $(0 \%)$ & 6 & & 14 & $(100 \%)$ & 0 & $(0 \%)$ & 14 & \\
\hline \multicolumn{13}{|l|}{ Clinical stage } \\
\hline $\mathrm{cT} 2$ & 25 & $(33.8 \%)$ & 49 & $(66.2 \%)$ & 74 & $<0.001$ & 15 & $(65.2 \%)$ & 8 & $(34.8 \%)$ & 23 & 0.08 \\
\hline cT3 & 29 & $(100 \%)$ & 0 & $(0 \%)$ & 29 & & 21 & $(100 \%)$ & 0 & $(0 \%)$ & 21 & \\
\hline $\mathrm{cT} 4$ & 14 & $(100 \%)$ & 0 & $(0 \%)$ & 14 & & 5 & $(100 \%)$ & 0 & $(0 \%)$ & 5 & \\
\hline \multicolumn{13}{|c|}{$\geq c T 3 b-T 4 a$ disease } \\
\hline No & 23 & $(31.9 \%)$ & 49 & $(68.1 \%)$ & 72 & $<0.001$ & 13 & $(61.9 \%)$ & 8 & $(38.1 \%)$ & 21 & $<0.001$ \\
\hline Yes & 45 & $(100 \%)$ & 0 & $(0 \%)$ & 45 & & 28 & $(100 \%)$ & 0 & $(0 \%)$ & 28 & \\
\hline \multicolumn{13}{|c|}{ Post-RC risk classification } \\
\hline High & 51 & $(68.9 \%)$ & 23 & $(31.1 \%)$ & 74 & 0.002 & 24 & $(88.9 \%)$ & 3 & $(11.1 \%)$ & 27 & 0.27 \\
\hline Low & 17 & $(39.5 \%)$ & 26 & $(60.5 \%)$ & 43 & & 17 & $(77.3 \%)$ & 5 & $(22.7 \%)$ & 22 & \\
\hline
\end{tabular}

$2(3.0 \%)$ patients and partial responses (pT1, pTa, pTis) were achieved in $12(17.6 \%)$ patients. Forty-one $(35.0 \%)$ patients, all of whom had high-risk features on final pathology, received adjuvant chemotherapy after RC.
Risk classification of patients who did receive NAC

Of the 49 patients treated with NAC, $43(87.8 \%)$ received cisplatin-based and six $(12.2 \%)$ received 
A. Cancer specific survival

Product-Limit Survival Estimates With Number of Subjects at Risk

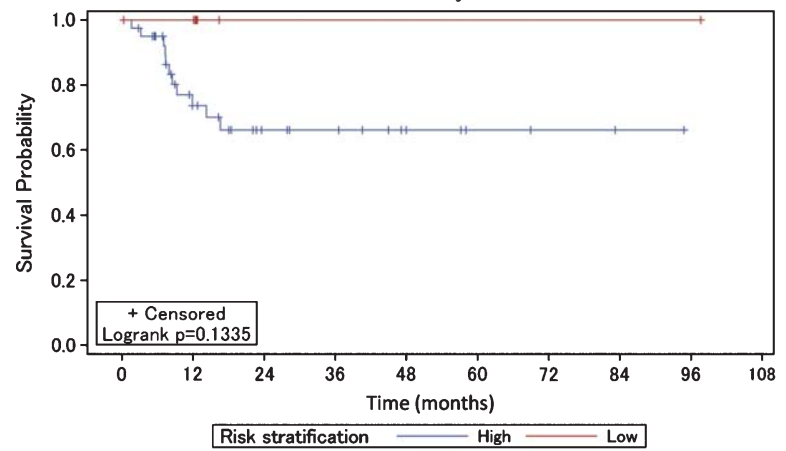

B. Overall survival

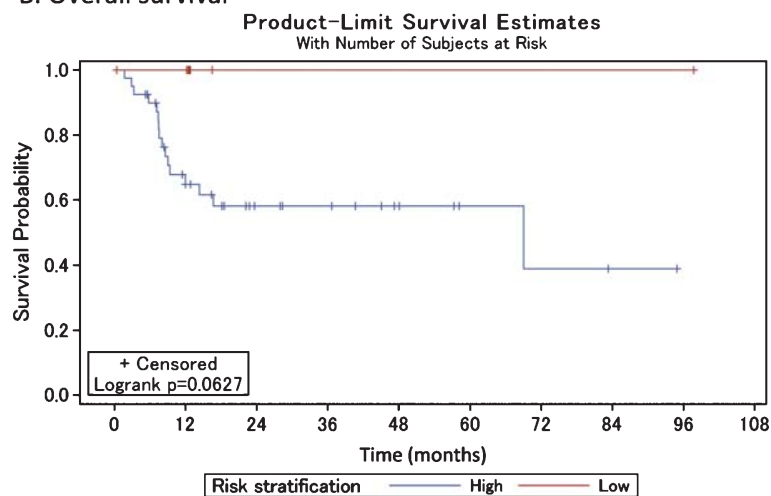

High 40

Fig. 3. Cancer-specific and overall survival among high- and low-risk patients who did not receive neoadjuvant chemotherapy.

Table 2

Pre- and post-operative risk stratification of patients according to whether or not they received neoadjuvant chemotherapy

\begin{tabular}{lcrc}
\hline NAC & Preoperative risk & No change & Change \\
\hline No & Low & $26(22.2 \%)$ & $23(19.7 \%)$ \\
& High & $51(43.6 \%)$ & $17(14.5 \%)$ \\
Yes & Low & $5(10.2 \%)$ & $3(6.1 \%)$ \\
& High & $24(49.0 \%)$ & $17(34.7 \%)$ \\
\hline
\end{tabular}

carboplatin-based regimens. Of note, utilization of NAC increased over time: 31 of 49 patients $(63.3 \%)$ were treated within the last five years of the time period analyzed in this report. Applying the MDACC model, we categorized $41(83.7 \%)$ patients as high risk and $8(16.3 \%)$ as low risk prior to treatment with
NAC and RC. High risk patients had a lower CSS and OS, though these results were not statistically significant (log-rank test $p=0.13$ and 0.06 for the two comparisons, respectively) (Fig. 4). There were too few events to reliably estimate age-adjusted hazard ratios of high- versus low-risk classification for cancer-specific and overall death. On final pathology after RC, $5(62.5 \%)$ low-risk patients remained classified as such, and $3(37.5 \%)$ were up-classified to high risk. Seventeen $(41.5 \%)$ high-risk patients were down-classified to the low-risk category, and $24(58.5 \%)$ remained high risk on final pathology. Complete pathologic responses (pT0) were seen in $13(26.5 \%)$ patients and partial responses (pT1, pTa, pTis) were seen in an additional10 (20.4\%) patients.

\section{A. Cancer specific survival}

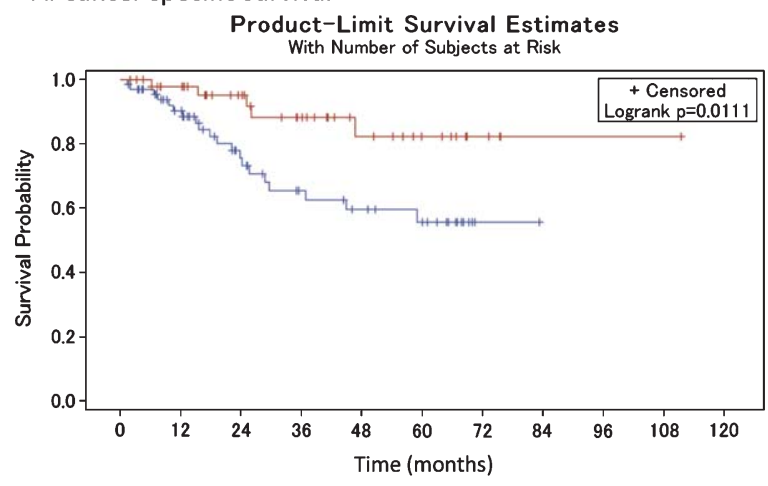

Risk stratification $=$ High - Low

\section{B. Overall survival}

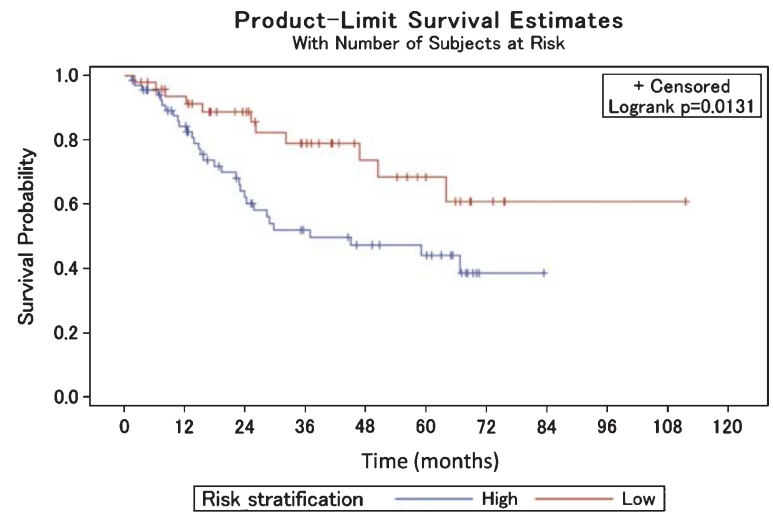

$\begin{array}{llllllllllll}\text { High } & 68 & 51 & 32 & 23 & 18 & 13 & 1 & 0 & & & \\ \text { Low } & 49 & 41 & 30 & 22 & 14 & 9 & 4 & 1 & 1 & 1 & 0\end{array}$

Fig. 4. Cancer-specific and overall survival among high- and low-risk patients who did receive neoadjuvant chemotherapy. 
Association between NAC use, CSS, OS, and post- $R C$ risk reclassification

Although the utilization of NAC was not statistically significantly associated with CSS or OS (log-rank test $p>0.05$ for both comparisons), it was associated with a 1.2 times increased odds $(95 \% \mathrm{CI}$ : 0.4 to 2.1 ) of post-RC reclassification from high to low risk on age-adjusted logistic regression.

\section{DISCUSSION}

Despite level I evidence from multiple phase III trials, cisplatin-based NAC in combination with RC in the treatment of muscle-invasive bladder cancer remains underutilized. The phase 2 prospective quality of care initiative of the Bladder Cancer Advocacy Network (BCAN) demonstrated increased utilization of NAC from $14 \%$ in the retrospective phase 1 portion of the study, to $47 \%$ by implementing simple procedures such as standard referral to a medical oncologist for consideration of a multimodal treatment regimen [11]. Preliminary data from the SWOG 1011 trial demonstrates similar utilization of NAC in $54 \%$ of patients, though with considerable variability between centers [12]. The most common reasons for patients to not receive NAC were patient preference $(33 \%)$, surgeon decision $(33 \%)$, and inadequate renal function (17\%). While SWOG 8710 reported a benefit of NAC for all pathologic stages, the survival advantage was most pronounced in patients with clinically non organ-confined disease [1]. In a separate study, patients with pathologic organ-confined disease had a recurrence-free survival of about $80 \%$ at five years [13]. It is a common perception among urologic oncologists that in this population NAC use would constitute overtreatment of surgically resectable organ-confined disease.

NAC is associated with a substantial toxicity profile, with the most common complications being granulocytopenia in $33 \%$ of patients and grade 3 or higher gastrointestinal complications in $17 \%$ of patients [1]. Despite these toxicities, the majority of patients recover full baseline performance status and are able to undergo RC after NAC [5]. In fact, the SWOG 8710 trial reported a similar proportion of patients undergoing $\mathrm{RC}$ regardless of the treatment arm to which they were assigned ( $81 \%$ for RC only versus $82 \%$ for NAC and RC), refuting the assertion that NAC use entailed a high risk of "missing" RC.

Another commonly stated concern of patients and physicians is the potential risk of delaying rather than entirely "missing" RC after NAC administration. Multiple authors have reported inferior outcomes for patients undergoing $\mathrm{RC}$ more than twelve weeks after the initial diagnosis (i.e., the date of their TURBT) $[14,15]$. Park et al. recently examined whether NAC delayed surgery and adversely affected survival. They found that the time intervals from TURBT to the initiation to NAC, from the initiation of NAC to RC, and from TURBT to RC were not associated with OS [16]. An important limitation was that their database did not capture patients who did not undergo RC due to disease progression or chemotherapy-related toxicities.

A risk-adapted strategy assumes that one can identify patients who are most likely to benefit from NAC and to spare those who may be at low risk for locally advanced or occult metastatic disease from potential NAC toxicity. The MDACC risk-stratification criteria proposed by Culp et al. mark an attempt to evaluate the predictive value of comprehensive clinical staging integrating pathologic data, information from cross-sectional imaging, and the physical exam [9]. They observed a lower five-year CSS (64.3\% vs. $83.5 \%)$ and OS (47\% vs. $64.8 \%$ ) for patients in the high-risk versus the low-risk group. Yet $49.2 \%$ of patients determined to be low risk were upstaged to the high-risk category after RC. Their findings were validated in the University of Southern California cohort, which showed similar five-year survival in both groups. Our results are consistent with these findings. We did observe a lower CSS and OS for patients in the high-risk category not treated with NAC prior to RC. Yet in patients not receiving NAC, we observed up-classification to the high-risk group in only $23(46.9 \%)$ patients.

The evaluation of clinical risk criteria does also rely on the clinical examination making this datapoint potentially operator dependent. It has been reported that clinical under-staging based on pathologic findings and pelvic EUA during TURBT is reported to occur in about $50 \%$ of patients $[17,18]$. Despite the EUA being a subjective test, a recent study found that $30.9 \%$ of patients have an EUA suggestive of pT3 disease [19]. The combination of cross-sectional imaging and EUA has a specificity of $89 \%$ and a negative predictive value of $74 \%$, making it a valuable test to rule out $\mathrm{pT} 3$ disease.

Computed tomography (CT) is the most commonly recommended imaging modality in the initial workup and is generally used to rule out distant metastasis as well as to identify grossly enlarged regional lymph nodes, both of which affect decision-making 
regarding multi-disciplinary treatment. However, CT has very poor performance characteristics for accurately predicting the $\mathrm{T}$ stage of the bladder tumor itself. In a series of 80 consecutive CT scans performed prior to RC, Paik et al. observed under-staging in $54.9 \%$ of patients and correct identification of extra-vesical spread only in $4.9 \%$ of patients [20]. Others have also reported significant inter-observer variability regarding the evaluation of extra-vesical tumor growth by CT [21].

Specific microscopic histopathologic findings may be associated with a more aggressive clinical course in otherwise stage-matched patients. A recent metaanalysis of 20 studies involving 10,663 patients concluded that LVI was associated with significantly worse recurrence-free and CSS [22]. From experience in other organs, we can assume that there may be a significant variance in the reporting of LVI [23]. Nonetheless, despite the potential inter-observer variability, Kunju et al. were able to demonstrate a substantial correlation between LVI observed in the TURBT and RC specimens (65\%) [24].

The micropapillary variant is a biologically aggressive form of high-grade urothelial cancer. A recent study demonstrated a relatively low inter-observer agreement for its identification between 14 genitourinary pathologists [25]. The most accurate diagnostic feature was the presence of multiple micropapillae in a single lacuna that could not be explained by retraction artifact (Fig. 1). Some have recommended early aggressive therapy for micropapillary urothelial carcinoma, advocating RC for cT1 disease and suggesting that this variant may not respond well to cisplatin-based chemotherapy [26]. However, others have reported chemo-sensitivity similar to that observed for conventional urothelial cancer [27].

Small-cell carcinoma has an aggressive biologic potential and is usually seen in combination with conventional urothelial carcinoma. The diagnosis is primarily histologic, and the criteria are similar to those used in other organ sites (i.e., solid sheet of cells with overlapping nuclei and scant cytoplasm, brisk mitotic activity, frequent apoptotic bodies, and necrosis). A study of 44 patients from the Mayo Clinic advocated that RC be the primary approach for treating non-metastatic cases of small-cell carcinoma of the bladder [28]. However, a more recent study of 107 patients concluded that RC did not improve prognosis in patients with non-metastatic bladder small-cell carcinoma and that conservative treatment (i.e., chemoradiation) should be considered [29].
The most important argument for the utilization of NAC is the higher number of pT0 patients post NAC and RC. The SWOG 8710 trial had a pT0 conversion rate from $15 \%$ to $38 \%$ when patients were treated with NAC. Similarly, a pT0 stage was achieved in $12 \%$ and $33 \%$ of patients treated without or with NAC, respectively, in the International Collaboration of Trialists/Bladder Cancer Working Party MVAC trial. An important observation in our analysis was the higher proportion of patients who converted from high to low risk after NAC and RC (41.5\%) versus after RC alone (25.0\%). While our sample size was not sufficient to demonstrate a significant difference in survival between risk categories, conversion between risk categories - NAC was associated with a 1.2 times increased age-adjusted odds (95\% CI: 0.4 to 2.1) of post-RC risk down-classification - may be a prognostic indicator. However, a prospective randomized evaluation of a risk-adapted management may be challenging in the future as NAC is appropriately established as a standard of care in cisplatin eligible patients and supported by multiple guidelines.

The value of pre-chemotherapy risk stratification may ultimately lie in the identification of patients who do not exhibit high-risk features on initial clinical staging and may therefore not benefit from NAC. Despite the inherent risk of upstaging, these patients still have better survival outcomes. While clinical staging will continue to be important in the initial evaluation of patients with bladder cancer, there is a growing body of evidence that molecular phenotyping may be helpful in the assessment of potential chemotherapy response. DNA-repair genes such as ERCC1 and ERCC2 have been reported to be associated with response to cisplatin $[30,31]$. Geneexpression profiling will likely receive more attention in the future for patient-specific risk stratification [32]. Plimack et al. have reported that alterations in DNA repair genes (ATM, RB1, and FANCC) appear to be associated with pT0 rates and OS in patients with muscle invasive bladder cancer who receive cisplatin-based NAC [33]. SWOG S1314 is designed to validate the COXEN score as a predictor of pT0 response to cisplatin-based NAC (NCT-02177695).

Our study has important limitations. It is a retrospective analysis of data from a single surgeon and two institutions. There was no prospective data collection specifically addressing the risk criteria discussed in the paper. Most important, the number of patients, especially in the cohort of patients receiving NAC, was rather small, limiting the power of the survival analyses. 


\section{CONCLUSIONS}

We observed improved CSS and OS in patients classified as low risk compared to high risk in the patients who did not receive NAC. NAC was associated with a 1.2 times odds of post-RC risk down-classification due to a higher conversion rate from high to low risk among patients treated with NAC compared to patients treated with RC alone. Whether a risk-stratified approach to NAC utilization is appropriate and oncologically safe requires prospective validation.

\section{CONFLICT OF INTEREST}

The authors declare no conflict of interest.

\section{REFERENCES}

[1] Grossman HB, Natale RB, Tangen CM, et al. Neoadjuvant chemotherapy plus cystectomy compared with cystectomy alone for locally advanced bladder cancer. N Engl J Med 2003;349:859.

[2] International Collaboration of T., Medical Research Council Advanced Bladder Cancer Working, P., European Organisation for, $\mathrm{R}$, et al. International phase III trial assessing neoadjuvant cisplatin, methotrexate, and vinblastine chemotherapy for muscle-invasive bladder cancer: Long-term results of the BA06 30894 trial. J Clin Oncol 2011;29:2171.

[3] Sherif A, Rintala E, Mestad O, et al. Neoadjuvant cisplatinmethotrexate chemotherapy for invasive bladder cancer Nordic cystectomy trial 2. Scand J Urol Nephrol 2002; 36:419.

[4] Rintala E, Hannisdahl E, Fossa SD. et al. Neoadjuvant chemotherapy in bladder cancer: A randomized study. Nordic Cystectomy Trial I. Scand J Urol Nephrol 1993;27: 355.

[5] Neoadjuvant cisplatin, methotrexate, and vinblastine chemotherapy for muscle-invasive bladder cancer: A randomised controlled trial. International collaboration of trialists. Lancet 1999;354:533.

[6] Petrelli F, Coinu A, Cabiddu M, et al. Correlation of pathologic complete response with survival after neoadjuvant chemotherapy in bladder cancer treated with cystectomy: A meta-analysis. Eur Urol 2014;65:350.

[7] deVere White RW, Lara PN Jr, Goldman B, et al. A sequential treatment approach to myoinvasive urothelial cancer: A phase II Southwest Oncology Group trial (S0219). J Urol 2009; 181:2476

[8] Millikan R, Dinney C, Swanson D, et al. Integrated therapy for locally advanced bladder cancer: Final report of a randomized trial of cystectomy plus adjuvant M-VAC versus cystectomy with both preoperative and postoperative M-VAC. J Clin Oncol 2001;19:4005.

[9] Culp SH, Dickstein RJ, Grossman HB, et al. Refining patient selection for neoadjuvant chemotherapy before radical cystectomy. J Urol 2014;191:40.
[10] Mitra AP, Bartsch CC, Bartsch G Jr, et al. Does presence of squamous and glandular differentiation in urothelial carcinoma of the bladder at cystectomy portend poor prognosis? An intensive case-control analysis. Urol Oncol 2014;32:117.

[11] Bochner F, Sperling, Mashni, Bajorin, Shah, Kamat, Steinberg, Stadler, Grubb, Kibel, Schoenberg, Black, Zlotta, Kassouf, Lerner, Lotan. Multi-institutional quality care initiative (QCI) to improve the care of patients with invasive bladder cancer (B1Ca). J Clin Oncol 2014;32 (suppl 4; abstr 298):2014.

[12] Lerner SP, LH, Svatek RS, Koppie TM, Alva AS, La Rosa FG, Bangs R, Pal SK, Siamak Daneshmand S, Kibel AS, Canter DJ, Tangen CM, Thompson IM. A Phase III Surgical Trial to Evaluate the Benefit of a Standard Versus an Extended Pelvic Lymphadenectomy Performed at Time of Radical Cystectomy for Muscle Invasive Urothelial Cancer: SWOG S1011 (NCT \#01224665). In: AUA Annual Meeting May, 2015, 2014

[13] Stein JP, Lieskovsky G, Cote R, et al. Radical cystectomy in the treatment of invasive bladder cancer: Long-term results in 1,054 patients. J Clin Oncol 2001;19:666.

[14] Mahmud SM, Fong B, Fahmy N, et al. Effect of preoperative delay on survival in patients with bladder cancer undergoing cystectomy in Quebec: A population based study. J Urol 2006; $175: 78$

[15] Fahmy NM, Mahmud S, Aprikian AG. Delay in the surgical treatment of bladder cancer and survival: Systematic review of the literature. Eur Urol 2006;50:1176.

[16] Park JC, Gandhi NM, Carducci MA. et al. A Retrospective Analysis of the Effect on Survival of Time from Diagnosis to Neoadjuvant Chemotherapy to Cystectomy for Muscle Invasive Bladder Cancer. J Urol 2016;195:880.

[17] Ficarra V, Dalpiaz O, Alrabi N, et al. Correlation between clinical and pathological staging in a series of radical cystectomies for bladder carcinoma. BJU Int 2005;95:786.

[18] McLaughlin S, Shephard J, Wallen E. et al. Comparison of the clinical and pathologic staging in patients undergoing radical cystectomy for bladder cancer. Int Braz J Urol 2007;33:25

[19] Rozanski AT, BC, McCoy JA, Green C, Grossman HB, Svatek RS, Shah JB. Is exam under anesthesia still necessary for the staging of bladder cancer in the era of modern imaging ? Bladder Cancer Journal Bladder Cancer 2015;1:91-96 91. DOI: $10.3233 /$ BLC-150006.

[20] Paik ML, Scolieri MJ, Brown SL, et al. Limitations of computerized tomography in staging invasive bladder cancer before radical cystectomy. J Urol 2000;163:1693.

[21] Tritschler S, Mosler C, Tilki D, et al. Interobserver variability limits exact preoperative staging by computed tomography in bladder cancer. Urology 2012;79:1317.

[22] Tian YF, Zhou H, Yu G, et al. Prognostic significance of lymphovascular invasion in bladder cancer after surgical resection: A meta-analysis. J Huazhong Univ Sci Technolog Med Sci 2015;35:646.

[23] Kryvenko ON, Epstein JI. Histologic criteria and pitfalls in the diagnosis of lymphovascular invasion in radical prostatectomy specimens. Am J Surg Pathol 2012;36:1865.

[24] Kunju LP, You L, Zhang Y, et al. Lymphovascular invasion of urothelial cancer in matched transurethral bladder tumor resection and radical cystectomy specimens. J Urol 2008; 180:1928.

[25] Sangoi AR, Beck AH, Amin MB, et al. Interobserver reproducibility in the diagnosis of invasive micropapillary 
carcinoma of the urinary tract among urologic pathologists. Am J Surg Pathol 2010;34:1367.

[26] Kamat AM, Dinney CP, Gee JR, et al. Micropapillary bladder cancer: A review of the University of Texas M. D. Anderson Cancer Center experience with 100 consecutive patients. Cancer 2007;110:62.

[27] Meeks JJ, Taylor JM, Matsushita K, et al. Pathological response to neoadjuvant chemotherapy for muscle-invasive micropapillary bladder cancer. BJU Int 2013;111: E325.

[28] Choong NW, Quevedo JF, Kaur JS. Small cell carcinoma of the urinary bladder. The Mayo Clinic experience. Cancer 2005; 103:1172.

[29] Pasquier D, Barney B, Sundar S, et al. Small Cell Carcinoma of the Urinary Bladder: A Retrospective, Multicenter Rare Cancer Network Study of 107 Patients. Int J Radiat Oncol Biol Phys 2015;92:904.
[30] Liu D, Plimack ER, Hoffman-Censits J, et al. Clinical Validation of Chemotherapy Response Biomarker ERCC2 in Muscle-Invasive Urothelial Bladder Carcinoma. JAMA Oncol, 2016.

[31] Hemdan T, SU, Malmström P.-U. ERCC1-negative tumors benefit from neoadjuvant cisplatin-based chemotherapy whereas patients with ERCC1-positive tumors do not results from a cystectomy trial database. EAU Annual Meeting 2014, 2014.

[32] Van Allen EM, Mouw KW, Kim P, et al. Somatic ERCC2 mutations correlate with cisplatin sensitivity in muscleinvasive urothelial carcinoma. Cancer Discov 2014;4:1140.

[33] Plimack ER, Dunbrack RL, Brennan TA, et al. Defects in DNA Repair Genes Predict Response to Neoadjuvant Cisplatin-based Chemotherapy in Muscle-invasive Bladder Cancer. Eur Urol 2015;68:959. 